\title{
Pushing the limits of EUV mask repair: addressing sub-10 nm defects with the next generation e-beam-based mask repair tool
}

\author{
Tilmann Heil $\odot,{ }^{\mathrm{a}, *}$ Michael Waldow, ${ }^{\mathrm{a}}$ Renzo Capelli, ${ }^{\mathrm{b}}$ Horst Schneider, ${ }^{\mathrm{a}}$ \\ Laura Ahmels, ${ }^{a}$ Fan Tu $\odot,{ }^{a}$ Johannes Schöneberg, ${ }^{a}$ and Hubertus Marbach ${ }^{\mathrm{a}}$ \\ ${ }^{\mathrm{a}}$ Carl Zeiss SMT GmbH, Rossdorf, Germany \\ ${ }^{\mathrm{b}}$ Carl Zeiss SMT GmbH, Oberkochen, Germany
}

\begin{abstract}
Mask repair is an essential step in the manufacturing process of extreme ultraviolet (EUV) masks. Its key challenge is to continuously improve resolution and control to enable the repair of the ever-shrinking feature sizes on mask along the EUV roadmap. The state-of-the-art mask repair method is gas-assisted electron-beam (e-beam) lithography also referred to as focused electron-beam induced processing (FEBIP). We discuss the principles of the FEBIP repair process, along with the criteria to evaluate the repairs, and identify the major contributions determining the achievable resolution. As key results, we present several high-end repairs on EUV masks including a sub-10-nm extrusion achieved with the latest generation of e-beambased mask repair tools, the MeRiT ${ }^{\circledR}$ LE. Furthermore, we demonstrate the corresponding repair verification using at-wavelength (actinic) measurements. () The Authors. Published by SPIE under a Creative Commons Attribution 4.0 Unported License. Distribution or reproduction of this work in whole or in part requires full attribution of the original publication, including its DOI. [DOI: 10.1117/1.JMM.20.3 .031013]
\end{abstract}

Keywords: EUV lithography; mask repair; focused electron-beam induced processing; gasassisted electron lithography; nanotechnology.

Paper 21011SS received Apr. 23, 2021; accepted for publication Aug. 19, 2021; published online Sep. 7, 2021.

\section{Introduction}

Extreme ultraviolet (EUV) lithography today is deployed to high-volume chip manufacturing. ${ }^{1-3}$ Several leading chip manufacturers already announced the delivery of first products created with EUV lithography. The key advantage of EUV lithography is its extendibility. The extremely short EUV light wavelength of $13.5 \mathrm{~nm}$ enables the continuation of Moore's law for many technology nodes ahead, ${ }^{4,5}$ which is a tremendous value for the entire semiconductor industry. To realize this, significant technology challenges must be mastered. In particular, the EUV light properties drive significant changes in core elements and tools of the chip manufacturing process. ${ }^{1-3}$ An illustrative example for such an innovation step is the photo mask. The entire working principle and design had to be revised. Firstly, EUV masks need to be operated in reflection and not in transmission as conventional deep ultraviolet (DUV) masks. The fact that EUV light is strongly absorbed by all materials not only requires that all manufacturing tools using EUV light operate in vacuum, it also implies that the incorporated optical elements are reflective to maximize system transmission.

Second, complex multi-layers had to be developed to coat the mask. ${ }^{6}$ For lithography process productivity reasons, it is essential to maximize the reflectivity of each optical element, including the mask. Creating such a high-reflectance surface, however, is again limited by the physical properties of EUV light. The refractive index of all materials at $13.5 \mathrm{~nm}$ is close to 1, and differences in between materials are small. ${ }^{7}$ Thus, creating a high-reflectance EUV mask operated under near normal incidence angles can only be achieved by multilayer coatings, making EUV masks highly complex structures.

*Address all correspondence to Tilmann Heil, tilmann.heil@ zeiss.com 
Third, the mask topography affects the aerial image. The height of the absorbers structuring the mask is significantly larger than the EUV wavelength. Consequently, electro-magnetic interaction of the EUV light with the mask topography becomes important for the imaging result on wafer. Understanding and optimizing this interaction is a field of ongoing research. ${ }^{8,9}$ Finally, the optimization of the mask absorber material is a very active field of research toward the next technology nodes. Some developments target to reduce the absorber thickness via so called high-k materials, i.e., materials with a large absorption, to reduce the mask 3D effects. Further activities target to improve imaging by phase shifting masks using low-n materials. ${ }^{10}$ All activities focus on improvements to increase the overall mask reflectivity, imaging contrast, and hence larger process windows.

Consequently, EUV masks are significantly more complex than their predecessors in DUV lithography. Their manufacturing complexity and value is increasing accordingly. This also means that the economic pressure not to lose masks during the manufacturing process is soaring. Thus, the ability to repair EUV masks is becoming technologically and economically increasingly important.

Even though the fabrication of EUV masks is extremely advanced, it is not perfect, i.e., yielding certain amounts of defects. As explained above the repair of such defects is practically a prerequisite for the profitable application of EUV masks. Whereas several technologies exist for the repair of mature technology photomasks such as, e.g., nanomachining or laser-based repair techniques, all these technologies run into physical limitations at shrinking feature sizes. ${ }^{11,12}$ Therefore, the repair of the most advanced features is currently solely based on gas-assisted electron-beam (e-beam) lithography methods. ${ }^{13-15}$ These can be subsumed as focused electron-beam-induced processing (FEBIP) techniques. ${ }^{16-19}$ The basic principle of FEBIP is the very local alteration of adsorbed precursor molecules or/and the substrate by the impact of electrons. ${ }^{17,20}$ More specifically, focused electron-beam induced etching (FEBIE) ${ }^{15,16}$ is used as a subtractive and focused electron-beam induced deposition (FEBID) $)^{18,19,21}$ as an additive technique. To do so, the focused e-beam is applied in the presence of certain precursor molecules thus triggering the local removal (FEBIE) or deposition (FEBID) of material. In a vivid picture, one might think of the focused e-beam as a pen and the precursor molecules as ink to draw on very small scales.

In this paper, we review the repair of EUV masks using FEBIP and demonstrate high-volume manufacturing ready solutions for EUV mask repair with the required high accuracies. In Sec. 2, we discuss the physical mechanisms determining the repair accuracy to of EUV masks and we describe the procedures and metrics to control the quality and success of mask repairs. In Sec. 3, we introduce the latest mask repair tool, the MeRiT ${ }^{\circledR} \mathrm{LE}$, available since 2021 to provide solutions for future EUV mask repairs. Furthermore, we present EUV mask repair results achieved with and corresponding verification results using the Aerial Image Measurement System $\left(\right.$ AIMS $\left.^{\circledR}\right)$ EUV tool. In particular, we demonstrate the repair of line extrusions smaller than $10 \mathrm{~nm}$ meeting the specifications of the next technology nodes. Finally, we summarize our results and present an outlook on future developments in EUV mask repair.

\section{EPE and Minimum Repair Size-What is Limiting Resolution}

A major challenge of the FEBIP-based repair process is to address smaller defects with decreasing critical dimensions (CD) on EUV masks. However, the underlying physics and chemistry of the repair process is considerably complex. On a fundamental level, we identify three main contributions: the size of the electron affected area of the mask, ${ }^{22-26}$ the properties of the precursor molecules in general ${ }^{20}$ and particularly the mask material, ${ }^{22,23,25}$ and finally the instrumental setup. Furthermore, each of the listed contributors can be broken down in several subtopics all influencing the achievable resolution.

For example, the size of the electron affected area is obviously determined by the size and shape of the e-beam impinging on the surface. However, even more importantly, the electron affected area strongly depends on secondary processes such as scattering events and the release of secondary electrons (SE). ${ }^{19,22,25-28}$ The spatial range of these effects drastically decreases with decreasing primary energy of the e-beam. Thus, the electron affected area depends on the shape 
and size of the e-beam, the energy and current density of the primary e-beam and the material properties of the mask, such as density, elemental composition, and morphology.

To initiate the repair process, the electrons must interact with an adsorbed precursor molecule. Usually, the repair process relies on electron induced dissociation of the precursor. This again is a rather complex process. For example, the cross section for electron induced dissociation is dependent on the actual electron energy and can be triggered by different processes like dissociative electron attachment or dissociative ionization to name just two. Conventional wisdom holds that cross section is low for higher electron energies and higher for low electron energies, which indicates that indeed SE play a decisive role in FEBIP.

In the next step, the properties of the dissociation products determine the course of the FEBIP process. In addition, also the physicochemical properties of the intact precursor, such as sticking coefficient or mobility on the mask surface, are to be considered for a full understanding of the repair process.

The last major contribution is the instrumental setup, which is probably the best accessible of the discussed. Thereby the tool stability as, e.g., beam position accuracy or inherent vibrations contribute to the repair performance. To deal with the complexity of the mask repair process, we follow a semiempirical approach in which the minimum repair size (MRS) is used as the quantity to describe the repair precision. As discussed above, a major contribution to the MRS is the size of the mask area that is exposed to primary, backscattered, and SE which in turn trigger the electron induced surface chemistry. As mentioned above, the size of the SE-distribution depends on the mask material, the energy of the impinging electrons, their spatial distribution given by the properties of the e-beam, and the relative movement between mask and electron source, i.e., the so-called jitter, which is a purely instrumental contribution. To address the main goal to decrease the MRS, a reduced jitter, a smaller SE distribution, and a smaller spot size of the electron source are obviously beneficial. While reducing the jitter is a matter of engineering excellence, the SE distribution and the e-beam spot size are fundamentally linked. Since the mask material is preset, an effective way to reduce the SE exit area is by minimizing the energy of the impinging primary electrons. ${ }^{29}$ A reduced electron energy, however, leads to an enlarged e-beam spot size in conventional scanning electron microscopes (SEM). We have quantified these trade-off behaviors with Monte Carlo simulations of the SE distribution via the CASINO software ${ }^{30,31}$ and with calculations of the e-beam spot size for different column types. ${ }^{27}$ Figure 1 summarizes the results showing an increased repair resolution may occur at lower electron voltages if the reduction of the SE cloud overcompensates the loss in imaging resolution of conventional SEMs. However, at small voltages the increasing imaging errors of the SEM may still dominate. Thus, SEM columns providing optimized e-beam resolution even at low landing energies are key modules for further

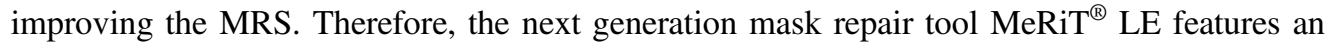
improved column with respect to its predecessor the MeRiT ${ }^{\circledR}$ neXT. Future column

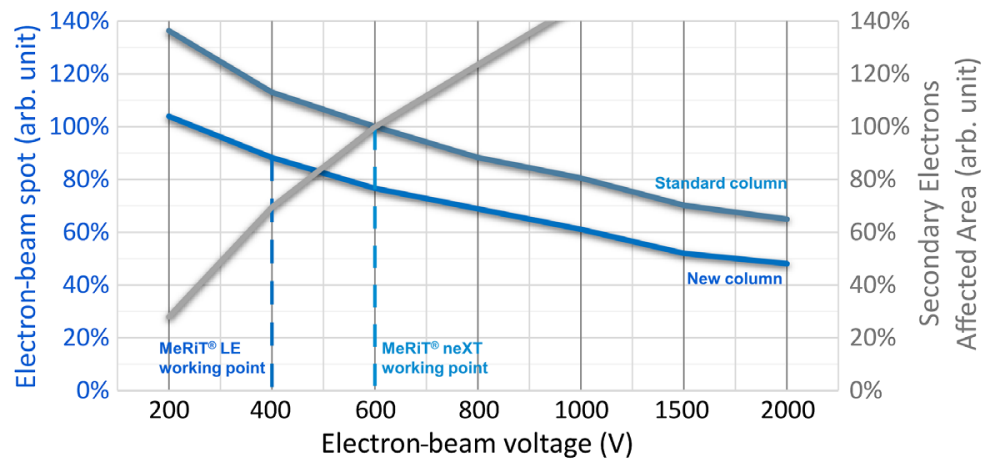

Fig. 1 The e-beam spot size for two different columns (light and dark blue) and relative spatial extension of the secondary electron distribution (gray) as a function of electron voltage. In addition, the working points of the MeRiT ${ }^{\circledR}$ neXT tool using a conventional column at $600 \mathrm{~V}$, and the MeRiT ${ }^{\circledR}$ LE tool using an optimized column at $400 \mathrm{~V}$ are indicated. Both, primary electron spot size, and secondary electron spot size have been reduced for the MeRiT ${ }^{\circledR}$ LE as compared to the MeRiT ${ }^{\circledR}$ neXT tool, thus enabling significantly smaller repair sizes. 
developments may even further extend the MRS roadmap to even lower electron voltages with aberration corrected e-beam columns.

The edge placement error (EPE) is another important criterion to describe how accurately the repair can be performed. The EPE is directly coupled to local CD errors ${ }^{32}$ that the repair process shall correct for. Hence, a repair process is successful when the positioning of the repaired edge relative to the target position stays within a well-defined range around the target edge. The EPE consists of an edge placement offset and noise on the repaired site, which is a result of the process chemistry. The edge placement offset is dominated by tool contributions, such as the exact determination of the defect location and shape, which defines the positioning of the e-beam during repair. Another important factor is the spatiotemporal alteration of this area in the SEM image during the repair, e.g., by charging or drift effects. Accordingly, the EPE reductions required by the semiconductor roadmap need continuous improvements of both, the tool design, and the repair processes.

\subsection{Repair and Verification}

The accuracy of the repair and hence the EPE can be determined by the position of the repaired edge relative to the target position as measured with the ZEISS AIMS ${ }^{\circledR}$ tools, an industry standard for defect disposition and repair verification of DUV as well as EUV masks. ${ }^{33}$ Within a mask shop the repair process and the subsequent printability verification process with an actinic imaging tool such as the AIMS ${ }^{\circledR}$ are closely connected since a mask repair is only deemed successful if the aerial image of the defective site on the mask is within specification. ${ }^{34} \mathrm{AIMS}^{\circledR}$ is an at-wavelength (actinic) metrology system specifically designed to emulate the aerial image formation as being done on exposure tools (e.g., ASML scanners). Figure 2 shows the core functionalities of the AIMS ${ }^{\circledR}$ EUV system in comparison to a wafer scanner. The illumination of the mask within the AIMS $^{\circledR}$ system is generated in an equivalent way compared to a scanner tool. While the optical design and components of the AIMS ${ }^{\circledR}$ tools may differ from the modules building up a scanner, they must be able to reproduce the same angular and spatial light intensity distribution locally within a limited light spot on the mask surface, assuring that the features on the mask experience (locally) the same light distribution they would experience on the scanner. After being collected by the numerical aperture (NA) on the mask side, the light is propagated in a projector lens (or mirror optics in the case of EUV) and the aerial image is magnified to be recorded with a CCD camera for post-evaluation. Not only the illumination of the mask must be emulated to achieve a complete and fully reliable printability statement, but the collection of diffraction orders as transmitted (DUV) or reflected (EUV) off the mask must be equivalent as on the scanner, to reproduce all relevant imaging effects which are significant for a thorough mask qualification, e.g., in the qualification of a repair. Thanks to this scanner equivalent aerial image generation process, and ability to measure through focus, $\mathrm{AIMS}^{\circledR}$ offers capabilities which go well beyond a printability statement, but allows to break down the components of an EPE and qualify different contributions deriving from the mask. This is one major advantage of such powerful metrology system, i.e., the capabilities to provide a full mask qualification, and

NXE: $33 \times 0-34 \times 0$ scanner

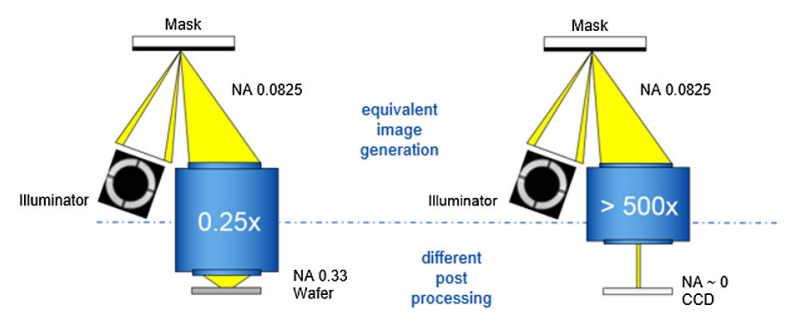

- $\quad$ IIMS $^{\circledR}$ EUV features scanner equivalent illumination and imaging

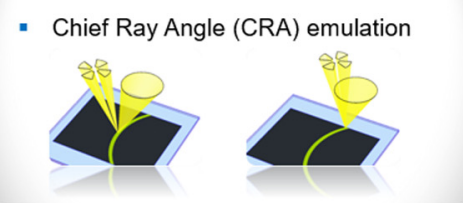

- Through focus aerial image

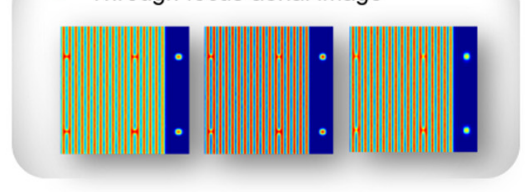

Fig. 2 AIMS ${ }^{\circledR}$ EUV core functionalities for actinic defect disposition and repair verification of EUV masks. 
therefore gaining tight control over an extensive metrology budget, without the need of printing one single wafer.

One of the challenges still open for having a seamless EUV production line is an inherent property of EUV light: each photon transports a considerable amount of energy when compared to $193 \mathrm{~nm}$ photons of DUV. As a consequence, the number of photons needed to develop a resist is much lower than for DUV lithography, giving rise to so-called stochastics failures on wafer, which to date are still a matter of concern in the community. In normal measurement mode, the AIMS $^{\circledR}$ EUV system itself uses an absolute intensity of light to locally illuminate a mask feature much larger compared to the ones in the scanner. However, being able to tune this light to match the flux seen by the single masks features locally on the mask, the AIMS ${ }^{\circledR}$ EUV is also able to capture the aerial image of a mask feature emulating the same amount of photon noise as produced in the scanner aerial image, before this gets absorbed into the resist and developed. The novel AIMS ${ }^{\circledR}$ EUV scanner stochastics emulation mode can be further employed in studying the statistical impact of mask error sources contributing to defectivity and/or EPE, being able to qualify defects or defect repairs from a statistical point of view, providing more insights into the effects such defects and different repairs have on the final wafer product. ${ }^{33}$ AIMS ${ }^{\circledR}$ Auto Analysis (AAA) ${ }^{35}$ is a powerful tool that automatically evaluates these light intensity maps and subsequently assesses the success of the repair without much overall need of user input.

\section{Repair Performance}

\subsection{Overview Photomask Repair Tool}

As detailed in a previous section, scaling trends in the semiconductor industry towards smaller technology nodes and feature sizes are continuing and first consumer products manufactured with EUV technology are already on the market. These developments lead to more strict technological requirements especially for the corresponding EUV photomasks in terms of repair accuracy, i.e., in particular MRS and EPE (see previous section). The current industry standard for high-end photomask repair tools is the $\mathrm{MeRiT}^{\circledR}$ neXT system. The next generation repair tool targeting the high-end photomasks of the upcoming technology nodes is the MeRiT ${ }^{\circledR}$ LE system. The MeRiT ${ }^{\circledR}$ LE offers a 35\% improved repair resolution compared to its predecessor and features a MRS of $10 \mathrm{~nm}$. It is designed to repair photomasks of the 5-nm technology node and beyond. ${ }^{36}$ With the MeRiT ${ }^{\circledR}$ LE transparent and opaque defects of many different geometries on DUV and EUV photomasks can be repaired successfully. The tool uses FEBIP technology and provides highest repair resolution. The MeRiT ${ }^{\circledR}$ LE makes use of a new e-beam column, which provides a decreased e-beam spot size at a given voltage compared to e-beam columns employed in predecessor MeRiT ${ }^{\circledR}$ tools. Furthermore, it is operated at a lower e-beam voltage of $400 \mathrm{~V}$, which reduces the area exposed to SE and shows a reduced jitter by improved tool damping. All these points together enable the repair of photomasks for future technology nodes with shrinking feature sizes (s. previous chapter). In Fig. 3, an image of the tool is depicted.

\subsection{High-End EUV Photomask Repair Results}

In this section, we present EUV mask repairs of transparent and opaque defects on a programmed defect mask (PDM) to verify the repair capabilities of the MeRiT ${ }^{\circledR}$ LE. The PDM is based on an industry standard EUV blank, i.e., using the same Mo-Si multi-layer and capping as EUV production masks and a binary Ta based absorber of $60 \mathrm{~nm}$ thickness. Please note that, unless stated otherwise, all dimensions are in mask dimensions, and not in wafer dimensions (Factor 4x for current EUV scanners). To demonstrate repair of opaque pattern defects, compact extrusions and bridge defects have been chosen. Broken-line defect types have been selected for clear defect repairs. All repairs have been verified by using AIMS ${ }^{\circledR}$ EUV actinic measurements, which provide a full emulation of the scanner imaging conditions. To quantify the measurement results, the AAA software has been used. Simply speaking, AAA automatically extracts edges from the recorded aerial images through focus thus allowing for quantitative Edge Placement and $\mathrm{CD}$ evaluations. Selected pattern sizes and defect types on the mask reflect decreased device 


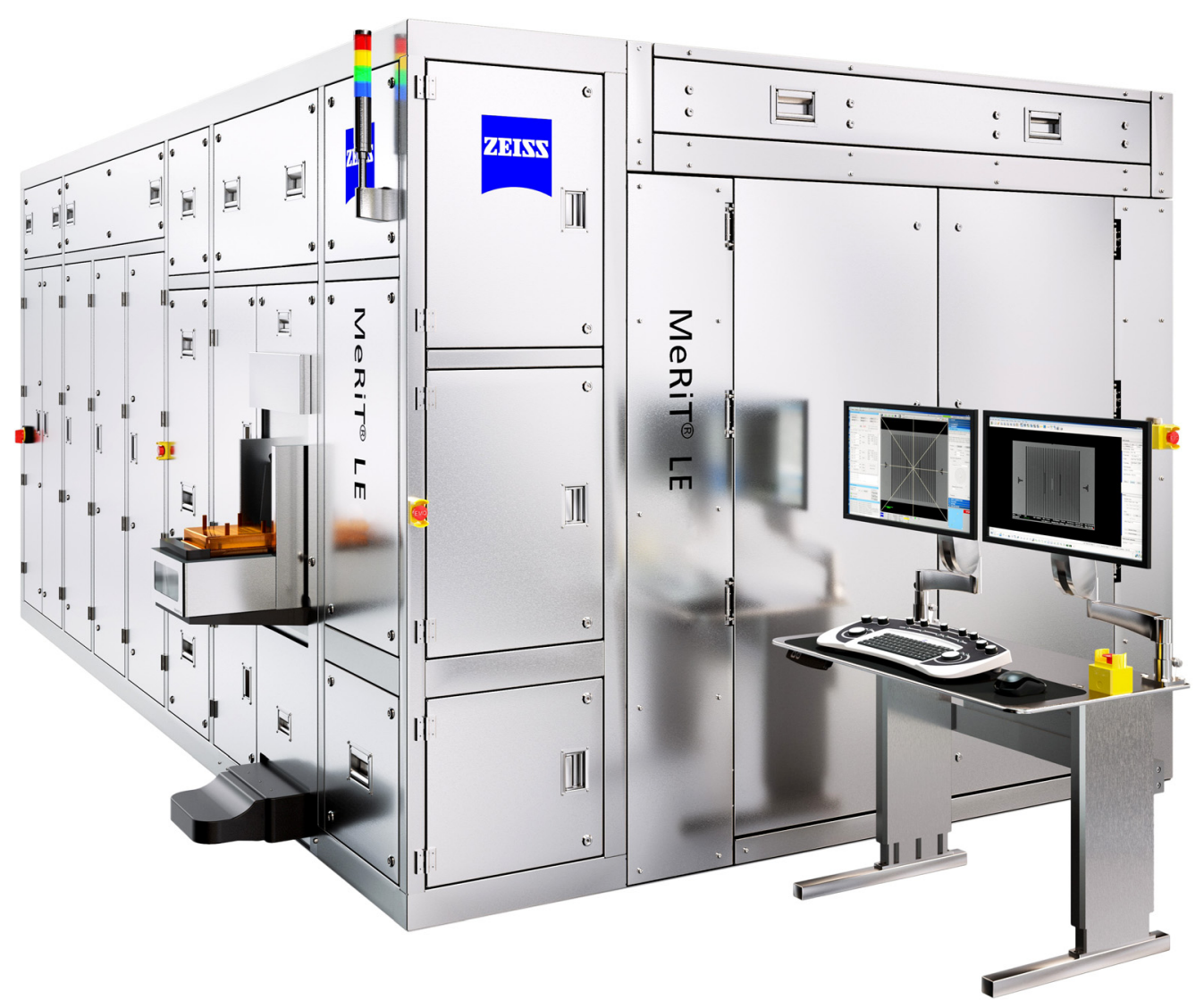

Fig. 3 View of the next generation photomask repair tool MeRiT ${ }^{\circledR}$ LE.

structures on high-end photomasks and increased complexity of pattern defects of the upcoming $5 \mathrm{~nm}$ technology node and beyond: Defects on EUV masks with feature sizes down to 60-nm half-pitch and extrusion widths of $9 \mathrm{~nm}$ in size have been successfully repaired.

For repair demonstration and verification, SEM Pre-Repair, SEM Post-Repair, and corresponding AIMS ${ }^{\circledR}$ EUV aerial images have been recorded. For all shown AIMS $^{\circledR}$ EUV measurements, NXE:3300 scanner Dipole aperture settings have been used. All results have been analyzed at best focus and through focus for various focal planes (FP). Here an FP of $\mathrm{FP}=-1$ describes a FP with a shift of $-1 \mu \mathrm{m}$ compared to the best focus plane $(\mathrm{FP}=0)$.

To illustrate what effect even small defects can have on the wafer print of high-end photomasks with shrinking feature sizes a small extrusion type defect with $500 \mathrm{~nm}$ in length, $9 \mathrm{~nm}$ in width, and a half-pitch size of $88 \mathrm{~nm}$ on mask has been analyzed. Figure 4(a) show the SEM image of the programmed defect and Fig. 4(b) the corresponding aerial image, acquired by AIMS $^{\circledR}$ EUV.

From the widened blue line in the center of Fig. 4(b), it becomes clear that the aerial image is significantly affected even by this tiny extrusion defect. To repair such small defects, the repair tool needs to provide an excellent MRS capability of better than $10 \mathrm{~nm}$ and a highly accurate repair edge placement. Subsequently, it is shown how this kind of small defects can be addressed by the MeRiT ${ }^{\circledR}$ LE system.

First investigated defect type on the EUV PDM represents the same as just discussed in the previous section, i.e. a small extrusion type defect with $500 \mathrm{~nm}$ in length, $9 \mathrm{~nm}$ in width, and a half-pitch size of $88 \mathrm{~nm}$ on mask. The repair is thus subtractive. Figures 5(a) and 5(b) show the SEM image of the defect before and after the repair, respectively, measured with the MeRiT ${ }^{\circledR} \mathrm{LE}$ system.

In Figs. 5(c) and 5(d) the post repair aerial image, acquired by AIMS ${ }^{\circledR}$ EUV and a corresponding detailed analysis of the $\mathrm{CD}$ after the repair carried out with the software AAA at best focus are shown. The black line in Fig. 5(d) represents the CD along the center of the defect site, 


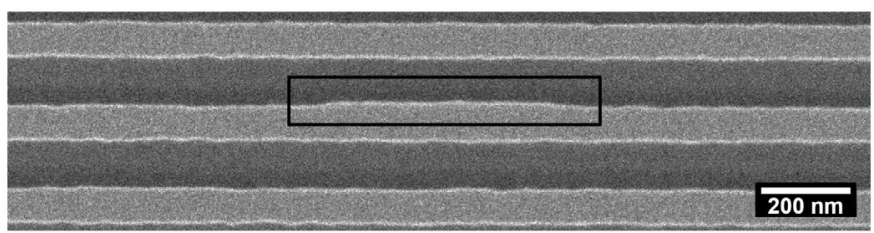

(a)

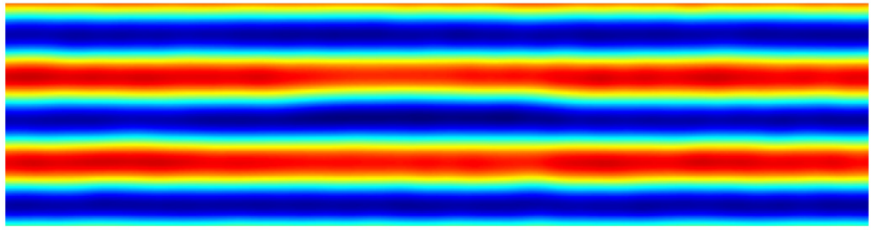

(b)

Fig. 4 (a) SEM image of a small extrusion type defect (9-nm width, 500-nm length) with 88-nm half-pitch on mask. (b) Aerial image by $\mathrm{AIMS}^{\circledR}$ EUV of the small extrusion defect. The defect can be clearly recognized in the center.

i.e., in "horizontal" direction indicated by the coordinate " $y$." The gray lines represent the corresponding CDs along the "unrepaired" neighboring reference "spaces," i.e., along the clear regions. From Fig. 5(d), it becomes evident that the CD variations along the center of the defect (black line) are below the $\mathrm{CD}$ variations of the reference, i.e., unrepaired regions (gray lines). A detailed analysis of the aerial image revealed for the repair a maximum CD deviation of $\Delta \mathrm{CD}_{\mathrm{MAX}}=3.3 \mathrm{~nm}$, whereas for the unrepaired reference regions a standard variation, representing $3 \sigma$-values, of $\Delta \mathrm{CD}_{\mathrm{REF}}=3.6 \mathrm{~nm}$ to the average $\mathrm{CD}$ reference value of $\mathrm{CD}_{\mathrm{REF}}=88$ has been determined.

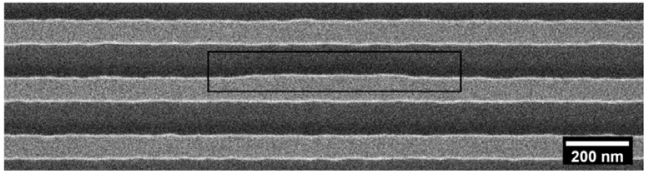

(a)

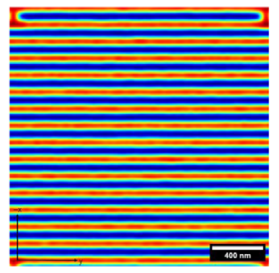

(c)

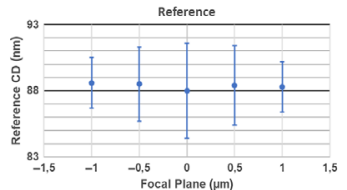

(e)

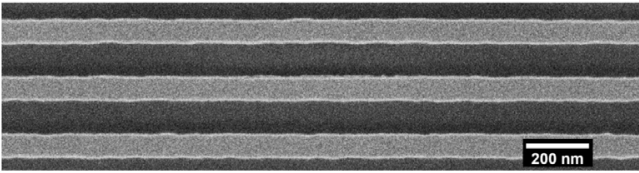

(b)

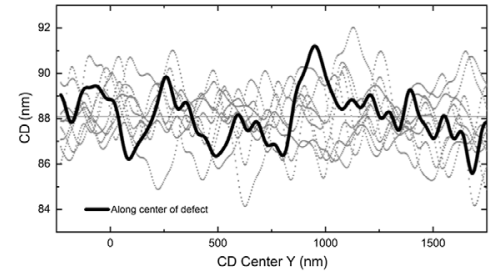

(d)

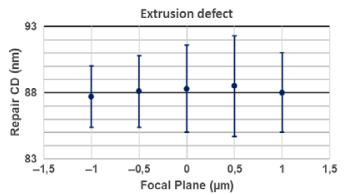

(f)

Fig. 5 (a) SEM Pre-Repair image of a small extrusion (9-nm width, 500-nm length) type defect with 88-nm half-pitch on mask. The dashed line indicates the desired absorber edge. (b) SEM PostRepair image of small extrusion defect. (c) Post-repair aerial image by AIMS ${ }^{\circledR}$ EUV of small extrusion defect. The repair is located in the center and is surrounded by four markers visible as red vertical lines in the very edges of the aerial image. (d) Post-repair aerial image analysis of the CD with AAA at best focus. The black line shows the CD along the repaired space. Gray lines show nearby reference structures, reflecting mask noise. (e) Aerial image analysis of the CDs at the reference sites in the vicinity of the repair for various FP. Blue points indicate the average values, and the bars represents the $3 \sigma$ deviations. (f) Post-repair aerial image analysis of the CDs of the repaired extrusion for various FP. Blue points indicate the average values, and the bars represents the $3 \sigma$ deviations. 


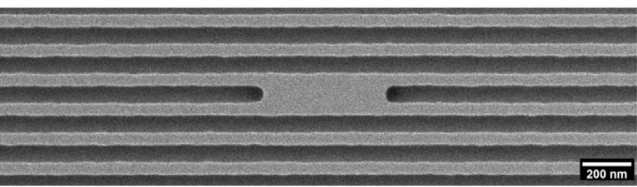

(a)

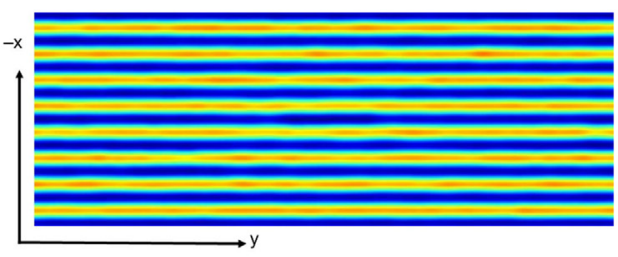

(c)

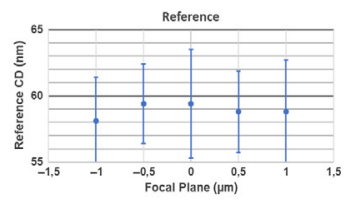

(e)

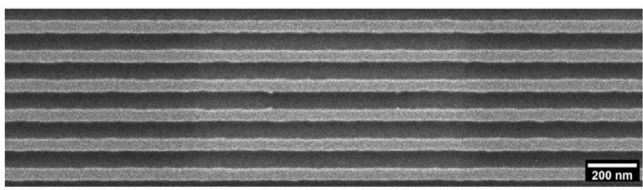

(b)

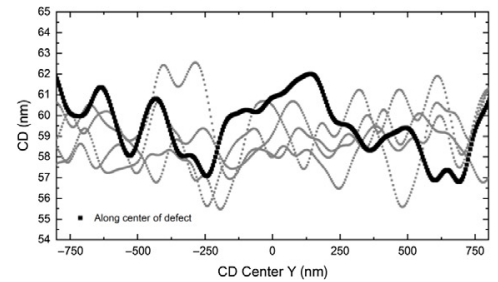

(d)

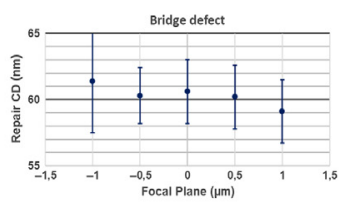

(f)

Fig. 6 (a) SEM Pre-repair image of a bridge (500 nm length) type defect with 60-nm half-pitch on mask. (b) SEM Post-repair image of bridge type defect. (c) Post-repair aerial image by AIMS ${ }^{\circledR}$ EUV of bridge type defect with 60-nm half-pitch on mask. (d) Post-repair aerial image analysis of the CD with AAA at best focus. The black line shows the CD along the repaired space. Gray lines show nearby reference structures, reflecting mask noise. (e) Aerial image analysis of the CDs at the reference sites in the vicinity of the repair for various FP. Blue points indicate the average values, and the bars represents the $3 \sigma$ deviations. (f) Post-repair aerial image analysis of the CDs of the repaired bridge defect for various FP. Blue points indicate the average values, and the bars represents the $3 \sigma$ deviations.

Figures 5(e) and 5(f) show the results of the through-focus analysis of the CD of the postrepair aerial image at various FP. In Fig. 5(e), the CDs for the reference structures, i.e., the unrepaired regions, in the vicinity of the repair, and in Fig. 5(f), the post-repair CD values are shown. Depicted CD values represent average values and corresponding deviations ( $3 \sigma$-values). From Figs. 5(e) and 5(f), it is evident that also for the other analyzed FP the CD deviations of the extrusion repair are about the variations of the reference CDs. This demonstrates that the MRS of the repair tool is better than $10 \mathrm{~nm}$. Furthermore, the average CD in reference site and repaired site are both well within $88 \mathrm{~nm}+/-1 \mathrm{~nm}$ demonstrating the Edge Placement capability of the repair tool. In total, these data verify the successful repair of the 9-nm extrusion with the MeRiT $^{\circledR}$ LE.

The second investigated opaque defect type on the EUV PDM is a bridge type defect with $500 \mathrm{~nm}$ in length and a half-pitch size of $60 \mathrm{~nm}$ on mask. The repair is thus subtractive. Figures 6(a) and 6(b) show the SEM image of the defect before the repair and the defect site after repair with the MeRiT ${ }^{\circledR}$ LE system. In Figs. 6(c) and 6(d) the post-repair aerial image, acquired by $\mathrm{AIMS}^{\circledR}$ EUV and a corresponding detailed analysis of the CD after the repair with the software AAA at best focus are depicted. The black line in Fig. 6(d) represents the CD along the center of the defect site, i.e., in horizontal direction indicated by the coordinate $y$. The gray lines represent the corresponding CDs along the unrepaired neighboring reference "lines," i.e., along the clear regions.

In Fig. 6(d), the $\mathrm{CD}$ variations along the center of the defect (black line) are below the $\mathrm{CD}$ variations of the reference regions (gray lines). A detailed analysis of the aerial image revealed for the repair a maximum $C D$ deviation of $\triangle C D_{\mathrm{MAX}}=2.6 \mathrm{~nm}$, whereas for the unrepaired reference regions a standard variation, representing $3 \sigma$-values, of $\Delta \mathrm{CD}_{\mathrm{REF}}=4.1 \mathrm{~nm}$ to the average $\mathrm{CD}$ reference value of $\mathrm{CD}_{\mathrm{REF}}=59.4 \mathrm{~nm}$ has been found. This indicates that at best focus the repaired regions appear even smoother than the original structures where the noise of the 


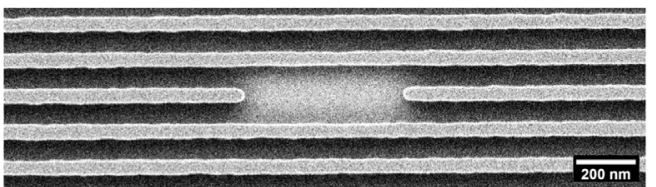

(a)

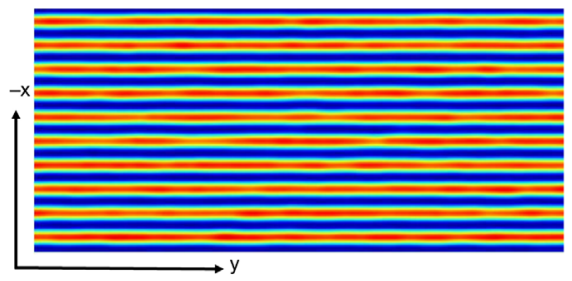

(c)

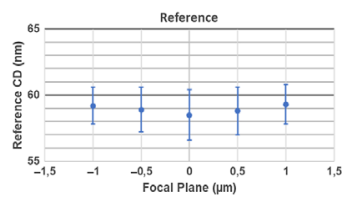

(e)

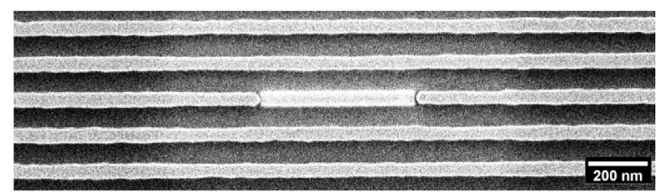

(b)

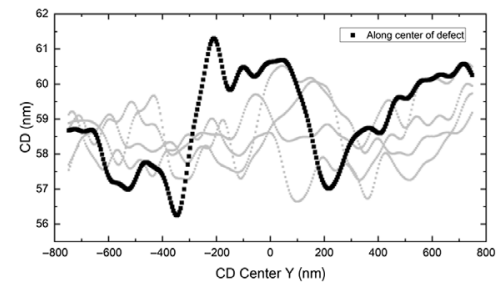

(d)

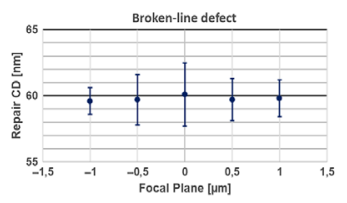

(f)

Fig. 7 (a) SEM Pre-Repair image of broken-line type defect (500-nm length) with $60 \mathrm{~nm}$ half-pitch on mask. (b) SEM Post-Repair image of broken-line defect. (c) Post-repair aerial image by AIMS ${ }^{\circledR}$ EUV of broken-line defect. (d) Post-repair aerial image analysis of the CD with AAA at best focus. The black line shows the CD along the repaired line. Gray lines show nearby reference structures, reflecting mask noise. (e) Aerial image analysis of the reference CDs in the vicinity of the repair for various FP. Average values and deviations ( $3 \sigma$ values) are given. (f) Post-repair aerial image analysis of the CDs of the repaired broken-line defect for various FP. Average values and deviations ( $3 \sigma$ values) are given.

structures is determined by the mask manufacturing process. Figures $6(\mathrm{e})$ and $6(\mathrm{f})$ show the results of the through-focus analysis of the $\mathrm{CD}$ of the Post-repair aerial image at various FP. From Figs. 6(e) and 6(f), it is evident that the CD deviations of the repair are comparable to or even below the $\mathrm{CD}$ deviations of the reference sites.

In addition to the extrusion and the bridge defect types, we have investigated clear defects on the EUV PDM using a broken-line type defect with $500 \mathrm{~nm}$ in length and a half-pitch size of $60 \mathrm{~nm}$ on mask, i.e. the repair in this case is additive. Figures 7(a) and 7(b) show the SEM image of the defect before the repair and a corresponding SEM image of the defect site after the repair with the MeRiT ${ }^{\circledR}$ LE system. In Figs. 7(c) and 7(d), the corresponding post-repair aerial image and the detailed analysis of the CD after repair carried out with AAA at best focus are shown. The black line in Fig. 7(d) shows the CD along the center of the defect site, i.e., in horizontal direction indicated by the coordinate $y$. The gray lines represent the corresponding CDs along the unrepaired neighboring reference lines, i.e., along the opaque regions.

In Fig. 7(d), the $C D$ variations along the center of the defect (black line) are about the $C D$ variations of the reference regions (gray lines).

Since the repair processes are still under optimization on the new tool, the edges of the deposited line do not yet fully close up on the mask feature line [Fig. 7(b)]. However, since the gaps are very small and the imaging process of the scanner acts as a low-pass filter, the post repair AIMS ${ }^{\circledR}$ EUV aerial image is only marginally affected [Fig. 7(c)]. Nevertheless, at the edges of the repair site (around $y= \pm 250 \mathrm{~nm}$ ) slightly higher CD deviations for the repair occur. Consequently, the maximum extracted $\mathrm{CD}$ deviation in that case is a bit higher compared to opaque defect types. A detailed analysis of the aerial image revealed a maximum $C D$ deviation of $\Delta \mathrm{CD}_{\mathrm{MAX}}=3.8 \mathrm{~nm}$ for the repair, whereas for the unrepaired reference regions a standard variation, representing $3 \sigma$ values, of $\Delta \mathrm{CD}_{\mathrm{REF}}=1.9 \mathrm{~nm}$ to the average $\mathrm{CD}$ reference value of $\mathrm{CD}_{\mathrm{REF}}=58.5 \mathrm{~nm}$ was determined. 
Figures 7(e) and 7(f) show the results of the through-focus analysis of the CD of the Postrepair aerial image at various FP. From Figs. 7(e) and 7(f), it appears that for the analyzed FP, the $\mathrm{CD}$ deviations of the repair are even lower than at best focus. Overall, the absolute $3 \sigma$-values of the repair CD are for all FP at a very low level with a highest $3 \sigma$-value of only $\Delta \mathrm{CD}_{3 \sigma}=2.4 \mathrm{~nm}$. It is worth mentioning that these $\mathrm{CD}$ deviations are the to our knowledge lowest values reported so far for a deposition repair at a half-pitch size of $60 \mathrm{~nm}$ on mask.

\section{Summary and Outlook}

In the work at hand, we report significant advances in EUV mask repair and verification equipment. Using the new MeRiT ${ }^{\circledR}$ LE system, EUV mask repairs of extrusions as small as $9 \mathrm{~nm}$ and of $60 \mathrm{~nm} \mathrm{L \& S}$ patterns on mask have been demonstrated and successfully verified

using the AIMS $^{\circledR}$ EUV tool and AAA software. These results demonstrate that the MeRiT ${ }^{\circledR}$ LE represents a valid EUV mask repair solution for the next technology nodes of the semiconductor roadmap.

Along with this development, two major trends are becoming apparent. First, the shrink of feature sizes keeps continuing and needs to be realized for the repair of EUV masks as well. This will be facilitated by the scaling of the repair mechanisms towards smaller repair sizes and better resolution described in this paper. The most important developments towards a reduction of the MRS are the reduction of the primary energy of the e-beam following a low-voltage roadmap, innovations in process chemistry and a higher stability of the tool itself. Secondly, the introduction of new EUV mask materials significantly improving the entire lithography process is imminent, and developments are ongoing throughout the industry. Overall, this is a very interesting new field of innovations with a high value potential. We speculate that we may see a similar development as for 193-nm lithography, where many different mask material options emerged over time. The MeRiT ${ }^{\circledR}$ focused e-beam technology inherently has a great flexibility to repair different material types including both DUV and EUV mask materials and is therefore well prepared to also support this trend. In conclusion, the MeRiT ${ }^{\circledR}$ LE provides the next generation EUV mask repair solution for the upcoming technology nodes further establishing mask repair as a cornerstone in the industry wide activities to support scaling and the continuation of Moore's law.

\section{Acknowledgments}

The authors would like to thank the Bundesministerium für Wissenschaft, Forschung und Wirtschaft (Federal Ministry of Science, Research and Economy), Award no. 16ESE0377K; and the ECSEL Joint Undertaking (JU) for funding under grant agreement No 826422. The JU receives support from the European Union's Horizon 2020 research and innovation program and Netherlands, Belgium, Germany, France, Romania, and Israel.

\section{References}

1. V. Bakshi, "EUV Lithography," SPIE Press, Bellingham, Washington (2018).

2. A. Pirati et al., "EUV lithography performance for manufacturing: status and outlook," Proc. SPIE 9776, 97760A (2016).

3. G. Tallents, E. Wagenaars, and G. Pert, "Lithography at EUV wavelengths," Nat. Photonics, 4(12), 809-811 (2010).

4. IEEE, "The international roadmap for devices and systems: 2020," 2020, https://irds.ieee .org/images/files/pdf/2020/2020IRDS_MM.pdf.

5. G. E. Moore, "Cramming more components on electronic circuits," Electronics 38(8), 114 (1965).

6. C. Montcalm et al., "Multilayer reflective coatings for extreme-ultraviolet lithography," Proc. SPIE 3331, 1-10 (1998). 
7. B. L. Henke, E. M. Gullikson, and J. C. Davis, "X-ray interactions: photoabsorption, scattering, transmission, and reflection at $E=50-30000 \mathrm{eV}, Z=1-92$," At. Data Nucl. Data Tables 54, 181-342 (1993).

8. J. T. Neumann et al., "Imaging performance of EUV lithography optics configuration for sub-9 nm resolution," Proc. SPIE 9422, 94221H (2015).

9. C. van Lare, F. Timmermans, and J. Finders, "Mask-absorber optimization: the next phase," J. Micro/Nanolithogr. MEMS, and MOEMS 19(2), 024401 (2020).

10. A. Erdmann et al., "Perspectives and tradeoffs of absorber materials for high NA EUV lithography," J. Micro/Nanolithogr. MEMS MOEMS 19, 041001 (2020).

11. T. Robinson et al., "Laser repair and clean of extreme ultraviolet lithography photomasks," Proc. SPIE 11518, 1151809 (2020).

12. M. J. Cadena, T. Robinson, and M. Klos, "Next generation nanomachining process technologies," Proc. SPIE 11518, 115180C (2020).

13. T. Liang et al., "Advanced photolithographic mask repair using electron beams," J. Vac. Sci. Technol. B 23(6), 3101-3105 (2005).

14. K. Edinger et al., "Electron-beam-based photomask repair," J. Vac. Sci. Technol. B 22(6), 2902-2906 (2004).

15. K. Edinger et al., "Application of electron-beam induced processes to mask repair," Proc. SPIE 5130, 1-8 (2003).

16. S. J. Randolph, J. D. Fowlkes, and P. D. Rack, "Focused, nanoscale electron-beam-induced deposition and etching," Crit. Rev. Solid State Mater. Sci. 31(3), 55-89 (2006).

17. I. Utke and A. Gölzhäuser, "Small, minimally invasive, direct: electrons induce local reactions of adsorbed functional molecules on the nanoscale," Angew. Chem. Int. Ed. 49(49), 9328-9330 (2010).

18. I. Utke, P. Hoffmann, and J. Melngailis, "Gas-assisted focused electron beam and ion beam processing and fabrication," J. Vac. Sci. Technol. 26(4), 1197-1276 (2008).

19. W. F. van Dorp and C. W. Hagen, "A critical literature review of focused electron beam induced deposition," J. Appl. Phys. 104(8), 081301 (2008).

20. P. Swiderek, H. Marbach, and C. W. Hagen, "Chemistry for electron-induced nanofabrication," Beilstein J. Nanotechnol. 9, 1317-1320 (2018).

21. M. Huth et al., "Focused electron beam induced deposition: a perspective," Beilstein J. Nanotechnol. 3(1), 597-619 (2012).

22. N. Silvis-Cividjian, C. W. Hagen, and P. Kruit, "Spatial resolution limits in electron-beaminduced deposition," J. Appl. Phys. 98, 084905 (2005).

23. W. F. van Dorp et al., "Approaching the resolution limit of nanometer-scale electron beaminduced deposition," Nano Lett. 5(7), 1303-1307 (2005).

24. L. van Kouwen, A. Botman, and C. W. Hagen, "Focused electron-beam-induced deposition of $3 \mathrm{~nm}$ dots in a scanning electron microscope," Nano Lett. 9(5), 2149-2152 (2009).

25. M.-M. Walz et al., "Investigation of proximity effects in electron microscopy and lithography," Appl. Phys. Lett. 100(5), 053118 (2012).

26. H. Plank et al., "Fundamental proximity effects in focused electron beam induced deposition," ACS Nano 6(1), 286-294 (2011).

27. J. E. Barth and P. Kruit, "Addition of different contributions to the charged particle probe size," Optik 101(3), 101-109 (1996).

28. W. F. van Dorp et al., "Focused electron beam induced processing and the effect of substrate thickness revisited," Nanotechnology 24, 345301 (2013).

29. K. Kanaya and S. Okayama, "Penetration and energy-loss theory of electrons in solid targets," J. Phys. D: Appl. Phys. 5(1), 43-58 (1972).

30. H. Demers et al., "Three-dimensional electron microscopy simulation with the CASINO Monte Carlo software," Scanning 33(3), 135-146 (2011).

31. D. Drouin et al., "CASINO V2.42 — a fast and easy-to-use modeling tool for scanning electron microscopy and microanalysis users," Scanning 29(3), 92-101 (2007).

32. J. Mulkens et al., "Overlay and edge placement control strategies for the $7 \mathrm{~nm}$ node using EUV and ArF lithography," Proc. SPIE 9422, 94221Q (2015).

33. R. Capelli et al., "Aerial image based metrology of EUV masks: recent achievements, status and outlook for the AIMS ${ }^{\mathrm{TM}}$ EUV platform," Proc. SPIE 10583, 1058311 (2018). 
34. K. Goldberg et al., "EUV actinic brightfield mask microscopy for predicting printed defect images," Proc. SPIE 9635, 963514 (2015).

35. G. Russell et al., "Automated defect disposition with AIMS AutoAnalysis," Proc. SPIE 10451, 1045113 (2017).

36. H. Schneider et al., "High-end EUV photomask repairs for $5 \mathrm{~nm}$ technology and beyond," Proc. SPIE 11518, 1151808 (2020).

Biographies of the authors are not available. 